\title{
Construction and Differential Screening of a cDNA Library Specific to Osmotic Stress of Haloxylon ammodendron Seedlings
}

\author{
Xiao-Cheng Jiang ${ }^{\dagger, s} *$, Xin-Hong Guo ${ }^{\dagger}$, Xiao-Ling Pan and Song-Quan Song ${ }^{\S}$ \\ ${ }^{\dagger}$ College of Life Science, Hunan Normal University, Changsha 410081, P. R. China \\ ${ }^{\star}$ Arid Ecological Environment Research Institute of Xinjiang University, Wulumuqi 830046, P. R. China \\ ${ }^{8}$ Xishuangbanna Tropical Botanical Garden, The Chinese Academy of Science, Menglun, Mengna 666303, Yunnan, P. R. China
}

Received 28 January 2004, Accepted 5 February 2004

A subtracted cDNA library specific to osmotic stress of Haloxylon ammodendron (Mey.) Bge seedlings was constructed by suppression subtractive hybridization (SSH) and T/A cloning. SSH was performed between two groups of $\boldsymbol{H}$. ammodendron seedlings, one was cultivated in Hoagland $(\mathrm{H})$ solution as a driver and the other group was treated with osmotic stress of the Hoagland solution by the addition of $400 \mathrm{mM}$ mannitol (M), as a tester. The library consisted of about 400 recombinant clones, with the average size being of $500 \mathrm{bp}$, ranging from $300 \mathrm{bp}$ to 1500 bp. Using a PCR-select differential screening kit, 100 recombinant clones were randomly chosen from the subtracted cDNA library and hybridized with forward,reverse subtracted and unsubtracted probes for two rounds. As a result, 21 positive clones specific to osmotic stress were obtained and some of them were verified by Northern blot analysis. The sequencing analysis of 6 positive clones and the following homology comparison to GenBank [blastx] non-redundant databases characterized that two sequences obtained in this experiment may contribute to novel drought-related genes.

Keywords: Haloxylon ammodendron, Osmotic stress, Sequence analysis, SSH, Subtracted cDNA library

\section{Introduction}

Drylands cover forty percent of the world's land surface (about 5.1 billion ha), and they are the habitat and source of livelihood for more than 1 billion people (World Atlas of

\footnotetext{
*To whom correspondence should be addressed. Tel: +86-691-8715237; Fax: +86-691-8715070

E-mail: jxclc@hunnu.edu.cn
}

Desertification, UNEP). Desertification affects seventy percent of the world's drylands, amounting to 3.6 billion ha or one fourth of the world's land surface. With the growth of population and the development of modern industry, desertification has become a growing concern all over the world. This concern is reflected in the Convention on Biological Diversity's drylands work program, and in the establishment of the UN Convention to Combat Desertification (CCD). To combat desertification and mitigate the effects of drought, it is very important to promote the effective and efficient use of existing plant resources.

The plant $H$. ammodendron grows in extremely dry or high salinity environments. On stabilized sandy soils and some lower slopes and basins, H. ammodendron "forests" are unique. These shrubs can reach over 10 feet high, which provides the desert with an unusual tall shrub component in a very xeric area. These "forests" also occur in many semiarid zones and they are important because they protect fragile soils from erosion and arrest sand movement; this plant has been used extensively in biological reconditioning of the desert (Bedunah and Schmidt, 2000; Zhu et al., 2003). Owing to the extreme tolerance of $H$. ammodendron to drought or salinity stress, it is referred to a super-xerophyte and acts as an important pioneer and constructive species in the Northwest arid region of China (Chen and Pan, 2000). It is assumed that molecules and compounds such as proline, glycine betaine and soluble sugars etc. synthesized and accumulated during desiccation play an important role in the protection of this plant from stress (Chen and Pan, 2000; Pan et al., 2000). However, at this time the molecular basis for these protective mechanisms is almost unknown. In this experiment, by means of suppression subtractive hybridization (SSH) (Diatchenko et al., 1996) and the relative techniques for screening differentially expressed cDNA clones, a cDNA library specific to osmotic stress of $H$. ammodendron seedlings has been constructed and two drought-related sequences were obtained. 


\section{Materials and Methods}

Plant materials $H$. ammodendron seeds were rinsed and cleaned by tap water and then placed on filter paper dampened by distilled water in Petri dishes at a constant temperature of $28^{\circ} \mathrm{C}$. After germination, seedlings were divided into two groups and transferred into new clean Petri dishes; one group was cultured in ordinary $1 \times$ Hoagland's solution $(\mathrm{H})$ and the other in $1 \times$ Hoagland's solution with the addition of $400 \mathrm{mM}$ mannitol (M). Both groups of seedlings had their roots immersed in their respective solutions. The culture was kept in an artificial climate chamber with $12 \mathrm{~h}$ of about $50 \mu \mathrm{mol} \mathrm{m} \mathrm{s}^{-2}$ light per day at 25 to $26^{\circ} \mathrm{C}$ for a week, during the time of culture the solution was renewed with the same concentration each day.

Total RNA isolation The buds of both types of seedlings were trimmed as materials for total RNA isolation. The procedures for the isolation were conducted according to the protocols of Rneasy Plant Mini Kit (Qiagen, Hilden, Germany).

Isolation and purification of poly $(\mathbf{A})^{+}$RNA From the total RNA obtained above, poly (A) ${ }^{+}$RNA was isolated and purified according to the protocols of Oligotex ${ }^{\mathrm{TM}}$ handbook (Qiagen).

Suppression subtractive hybridization (SSH) and construction of subtracted cDNA library The manipulation of the SSH we were concerned with was carried out in accordance with the user manual for Clontech PCR-Select ${ }^{\mathrm{TM}}$ cDNA Subtraction Kit.

The secondary PCR Products of SSH were inserted into pT-Adv vectors included in the AdvanTAge PCR cloning Kit (Clontech, Palo Alto, USA). The procedures were fulfilled largely in accordance to the introductions of the kit. However, prior to the insertion, the forward subtracted PCR cDNA mix was incubated for an extra $1 \mathrm{~h}$ at $72^{\circ} \mathrm{C}$ with additional dATP and Taq DNA polymerase (Invitrogen, Calsbad, USA) to ensure that most of the cDNA fragments contained 3'A overhangs. Otherwise, approximately $100 \mathrm{ng}$ PCR-amplified cDNA were ligated into $50 \mathrm{ng}$ vectors and the recombinants were then transformed into TOP10F' $E$. coli competent cells. Next, the transformed bacteria were plated onto $22 \times 22 \mathrm{~cm}$ agar plates with $100 \mu \mathrm{g} / \mathrm{ml}$ ampicillin, $100 \mu \mathrm{M}$ IPTG and $50 \mu \mathrm{g} / \mathrm{ml} \mathrm{X-Gal.} \mathrm{The} \mathrm{plates} \mathrm{were} \mathrm{incubated} \mathrm{at} 37^{\circ} \mathrm{C}$ for about $26 \mathrm{~h}$ until small colonies were visible, and then the plates were transferred into the refrigerator of $4^{\circ} \mathrm{C}$, a temperature at which the blue/white staining could be distinguished more clearly. The white colonies were the hypothetical $E$. coli cells including recombinant clones, and they were selected for further analysis by plasmid isolation, PCR, or sequencing.

Screen differentially expressed cDNA clones from the subtracted library A total of 400 hypothetical recombinant clones were picked out and then incubated in the standard 96-well microtitre plates containing LB medium with $100 \mu \mathrm{g} / \mathrm{ml}$ ampicillin. The incubation was done overnight on a rotary shaker at $37^{\circ} \mathrm{C} .1 \mathrm{ml}$ bacterial culture of each clone was used in the PCR amplification for cloning the target insert in $20 \mu \mathrm{l}$ reactions containing $2 \mu \mathrm{l} 10 \times$ PCR reaction buffer, $0.6 \mu \mathrm{l}$ Nested Primer $1,0.6 \mu \mathrm{l}$ Nested Primer $2 \mathrm{R}, 0.4 \mu \mathrm{l} 10 \mu \mathrm{M} \mathrm{dNTP} \operatorname{mix}$ (Clontech). The reaction was done under the following conditions: $94^{\circ} \mathrm{C}$ for $30 \mathrm{~s}, 23$ cycles at $\left(94^{\circ} \mathrm{C}\right.$ for
$30 \mathrm{~s}, 68^{\circ} \mathrm{C}$ for $3 \mathrm{~min}$ ). After amplification, four clones were randomly selected by taking $5 \mu \mathrm{l}$ for each one for electrophoresis on a $2 \%$ agarose/EtBr gel in $1 \times$ TAE buffer. The visible PCR product corresponded to the cDNA insert.

For screening of the subtracted library, $5 \mu \mathrm{l}$ of PCR product from each of the above PCR amplification samples were denatured by adding $5 \mu \mathrm{l}$ of freshly made $0.6 \mathrm{M} \mathrm{NaOH}$ to them in a 96-well plate. Then, 1-2 $\mu \mathrm{l}$ of the denatured product was blotted in duplicate onto nylon membranes and four nylon membranes with identical blots were prepared simultaneously. They were incubated overnight at $42^{\circ} \mathrm{C}$ in the presence of $100 \mu \mathrm{g} / \mathrm{ml}$ heat-denatured ultrasonictreated salmon sperm DNA, and hybridization was performed in $5 \mathrm{ml}$ of hybridization solution (Clontech) containing $5 \times 10^{7} \mathrm{cpm}$ per $100 \mathrm{ng}$ of cDNA probes prepared respectively by forward or reverse subtracted or unsubtracted cDNA (driver/tester). The membranes were washed with $2 \times$ SSC and $0.5 \%$ SDS four times at $68^{\circ} \mathrm{C}$, each washing time lasted for $20 \mathrm{~min}$, then this was followed by two high stringency washings with $0.2 \times \mathrm{SSC}$ and $0.5 \%$ SDS at $68^{\circ} \mathrm{C}$, and each washing time was also for $20 \mathrm{~min}$. Then each membrane was sealed between two pieces of semi-permeable filter and exposed to Kodak BioMax MR films overnight on an intensifying screen at $80^{\circ} \mathrm{C}$.

Northern blot analysis Two $\mu \mathrm{g}$ total RNA for each lane of driver and tester were used for electrophoresis on $1.0 \%$ formaldehyde agarose gel, then the samples were transferred onto a piece of nylon membrane. Differentially expressed candidate cDNAs were firstly amplified by PCR as described above and then labeled with $\left[\alpha-{ }^{32} \mathrm{p}\right]$ $\mathrm{dCTP}$ by random priming and they were used as probes for Northern blot hybridization. The following procedures were performed in accordance with the protocols for the Northern blot hybridization (Sambrook et al., 1989).

cDNAs sequencing and sequence analysis Individual recombinant clones containing differentially expressed cDNAs that were grown in Terrific broth were used to prepare plasmids for DNA sequencing. DNA sequencing was carried out by Beijing Saibaisheng Biotech Company Limited using an ABI Dyedeoxy Terminator Cycle Sequencing apparatus (ABI Prism 377 DNA sequencer, Applied Biosystems, Fostercity, USA). The sequences of the cDNA inserts were compared with the GenBank non-redundant TRANSLATED query-PROTEIN databases [blastx] after stripping out the vector and primer sequences.

\section{Results}

Preparation of total RNA, poly $(\mathrm{A})^{+}$RNA, and PCR-select cDNA subtraction (SSH) The total RNA samples prepared from $H$. ammodendron seedings under normal physiological condition and osmotic stress, were shown in Fig. 1A. The two samples ratio of $\mathrm{A}_{260} / \mathrm{A}_{280}$ in $10 \mathrm{mM}$ Tris- $\mathrm{HCl}, \mathrm{pH} 7.5$ is 1.91 and 1.92, respectively, which indicated the total RNA samples are of high quality. From the total RNA, their poly $(\mathrm{A})^{+}$RNA was isolated and purified.

According to the principle of the SSH method, a series of molecular events were carried out step by step up to the end of 


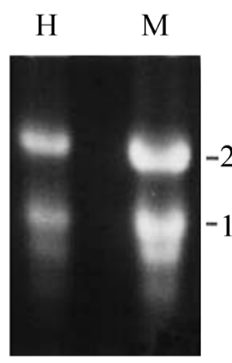

A

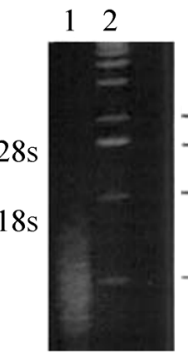

B

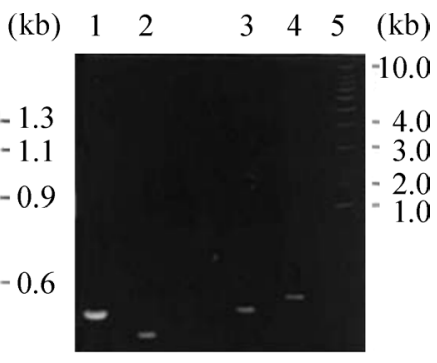

$\mathrm{C}$
Fig. 1. Electrophoresis analyses of the total RNA of the driver (A, lane $\mathrm{H})$ and the tester (A, lane $\mathrm{M})$, and the secondary PCR products of subtracted tester cDNAs $(\mathrm{B}$, lane 1$)$ and cDNA inserts in pT-Adv vectors amplified by site-specific PCR (C, lanes 1, 2, 3, and 4); Lane 2 of B and lane 5 of $\mathrm{C}$ indicated $\phi X 174$ DNA/Hae III-digested size markers and $1 \mathrm{~kb}$ DNA ladder, respectively.

the secondary PCR, and the PCR products of the subtracted tester cDNAs were analyzed by electrophoresis and looked like a smear without distinct bands (Fig. 1B, lane 1).

Construction and characterization of the subtracted cDNA library In our experiment, about 400 individual recombinants were yielded. To evaluate the recombining efficiency and measure the length of the cDNA inserts, from which four individual white bacterial colonies were randomly picked out and grown in LB-Amp medium overnight, the cDNA inserts were amplified by PCR and analyzed by agarose gel electrophoresis. Fig. 1C showed that all of four clones contained DNA inserts, each with an average size of approximately $500 \mathrm{bp}$. The results demonstrated that each PCR product corresponded to a cDNA insert.

Differential screening of the subtracted cDNA library Differential screening to the subtracted cDNA library was performed by cDNA dot blots of the PCR products, the partial results are shown in Fig. 2. In our experiment, from 100 recombinant vectors chosen randomly, 21 of them were identified as hybridizing with the forward-subtracted and unsubtracted tester probes. But they didnt hybridize or they very weakly hybridized with the reverse-subtracted and unsubtracted driver probes, Generally, the clones that hybridized only with the forward-subtracted tester probe were strong candidates for differentially expressed cDNA clones specific to osmotic stress. The clone $\mathrm{C} 4$ was such a clone, which typically had low-abundance transcripts and then was enriched during the subtraction. Those like clone C7 hybridizing with the forward subtracted and unsubtracted tester probes, but not with the reverse subtracted or unsubtracted driver probes, always corresponded to differentially expressed genes. Those clones having no detectable hybridization signal from either of the subtracted and unsubtracted probes usually represented the extreme lowabundance transcripts or false positive recombinants, such as clone A4. Those like clone B10, hybridizing equally with both subtracted probes and unsubtracted probes, were the most commonly expressed clones.

Northern blot analysis to the differentially expressed candidate cDNAs By Northern blot analysis of the probes prepared from 8 randomly selected clones (the putative osmotic stress-related candidates by differential screening as shown in Fig. 2), one half of them showed positive hybridization signals. Only 2 clones among them hybridized to the tester total RNA (Fig. 3D and 3E), 2 clones could hybridize with the total RNA of the tester and with the drivers as well, but they had the stronger signals to the former (Fig. $3 \mathrm{~B}$ and $3 \mathrm{C}$ ). Therefore at least $50 \%$ of the candidate clones could be confirmed as harboring specific or differentially expressed cDNA to osmotic stress. While the other four candidate clones had no signals to the two total RNA samples (not shown), maybe these clones expressed abundance was too low to be checked by Northern blot hybridization.

Sequencing and homology analysis of the specific or differentially expressed cDNAs Of the 21 identified clones specific or differentially expressed to osmotic stress, 6 clones with positive hybridization signals to the total tester RNA by Northern blot analysis, including the 4 clones shown in Fig. 3, were subjected to sequencing and homology analysis. As seen on sequencing, 2 different putative sequences related to osmotic stress were obtained, ( 2 clones represent Sequence I, and all of the other 4 clones contain Sequence II). The fact that more than one clone contains the same sequence of cDNA especially implies that these two sequences should have multi-copies in the genome of $H$. ammodendron. If this feature of cDNA sequences of a xerophyte is associated with drought tolerance, then it certain requires further exploration. The results of these two sequences as compared with the GenBank non-redundant TRANSLATED query-PROTEIN databases [blastx] were listed in Fig. 4:

Homology analysis indicated that both of the above 2 sequences did not completely match to any known genes or sequences in the GenBank databases, so it is assumed that they represent novel drought-related genes. On the other hand, Sequence I from nucleotide 19-339 matches to the nucleotide sequence 424-530 of a glycine-rich protein Arabidopsis thaliana; Sequence II, whose nucleotide 22-294 reversely matches to the nucleotide sequence $94-4$ of a putative senescence-associated protein of snow pea pods (Pisum sativum L. var saccharatum) (Pariasca et al., 2001). Moreover, by comparison Sequence II was shown to have a defect of 6 nucleotides for the amino acids tryptophan and tyrosine at nucleotide sites 127-129 and 160-162, respectively. From the results of the present work, we also speculate that the glycinerich protein (gi|22328751|ref|NP_193498.2|) of the model plant Arabidopsis thaliana and the putative senescence-associated protein of snow pea pods (gi|13359451|dbj|BAB33421.1|) may have roles in the plants response to drought tolerance. 


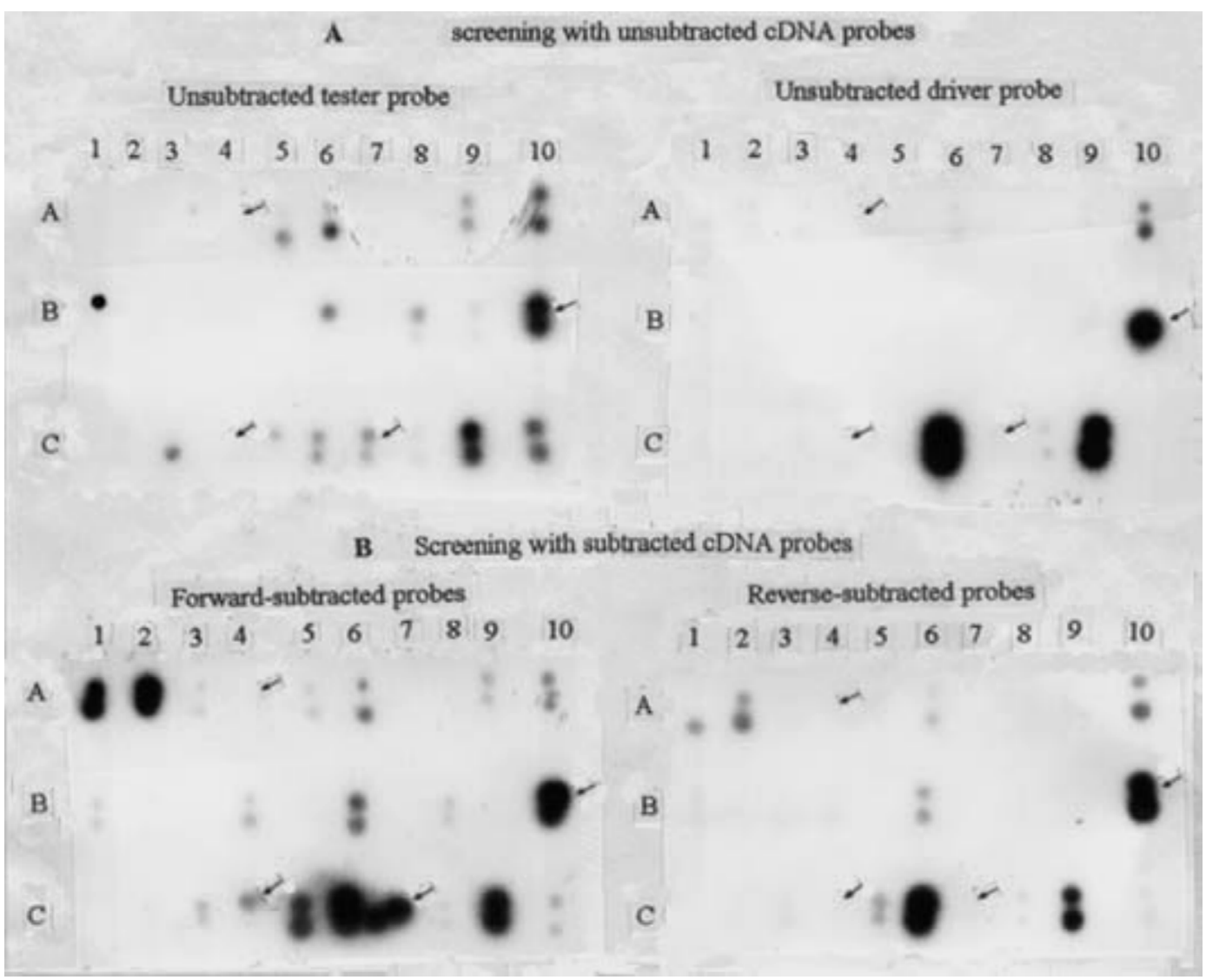

Fig. 2. Differential screening results. Panel A, cDNA Dot blots hybridized with unsubtracted cDNA probes made from tester (M) and driver $(\mathrm{H})$ poly $(\mathrm{A})^{+}$RNA; Panel B, Dot blots hybridized with cDNA probes made from forward-subtracted cDNA (M, tester; H, driver) and reverse-subtracted cDNA (M, driver; $\mathrm{H}$, tester). As shown by the arrows, A4 had no detectable signal hybridized with four types of probe; B10 almost equally hybridized with four types of probe; C4 only hybridized with forward-subtracted probe; C7 only hybridized with unsubtracted tester probe and forward-subtracted probe.
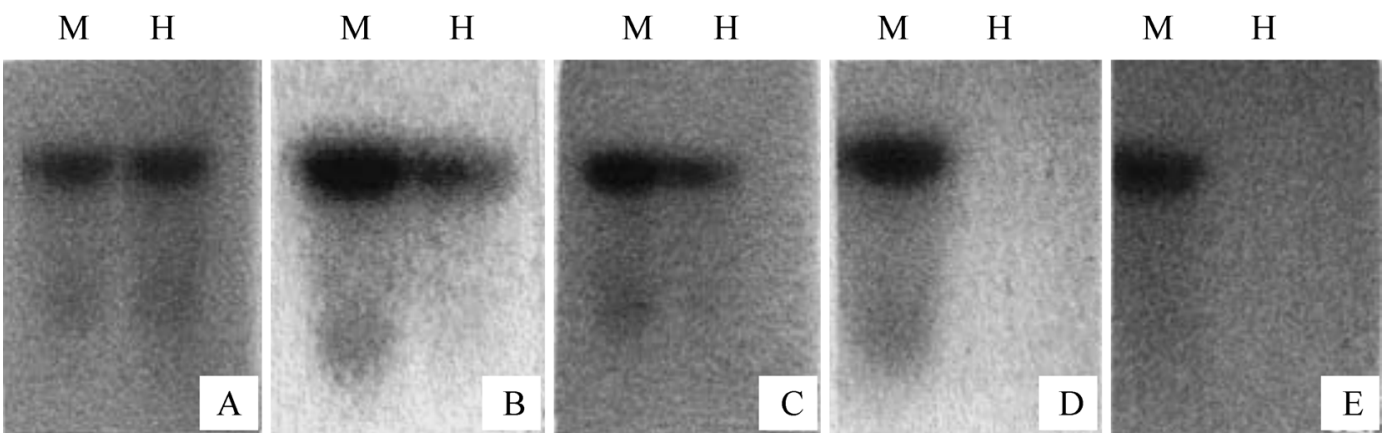

Fig. 3. Northern blot hybridization of tester $(\mathrm{M})$ and driver $(\mathrm{H})$ with the probes made from the cDNA clones (as Fig. 2, C9) equally expressed in the tester and the driver (A) or from the cDNAs (as Fig. 2, C4 and C7) specifically or differentially expressed in the tester (B-E) (partial results). The result shown in (A) indicated the same quantity of total RNA samples of tester (M) and driver (H) was loaded.

\section{Discussion}

Upon exposure to drought and high salinity stress, plants exhibit a wide range of responses at the molecular, cellular and whole-plant levels (Bohnert and Sheveleva 1998; Hasegawa et al., 2000). Desiccation-tolerant plants provide excellent models for genetic and biochemical studies (Bartels et al., 1990; Ingran and Bartels, 1996). These plants can adapt to an environment of extreme dehydration by morphological and developmental changes (e.g. life cycle, inhibition of shoot growth and enhancement of root growth), adjustment in ion transport (such as uptake, extrusion and sequestration of ions), 


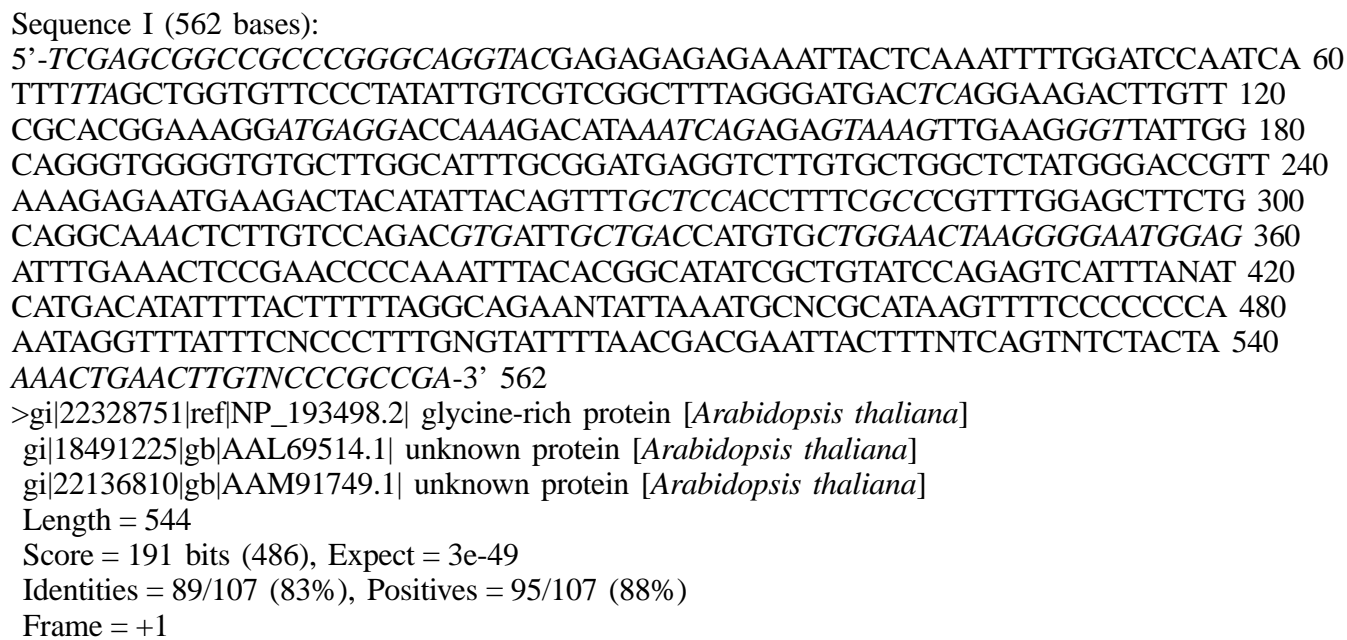

Query: 19 RYEREKLLKFWIQSFLAGVPYIVVGFRDDSGRLVRTERMRTKDINQRVKLKGYWQGGVCL 198 R+EREKLLKFWIQSF+AGVPYIVVGFRDD GRLVRTER+T+DI R+LK YWQGGVCL

Sbjct: 424 RFEREKLLKFWIQSFVAGVPYIVVGFRDDGGRLVRTERLTTRDIAHRARLKNYWQGGVCL 483

Query: 199 AFADEVLCWLYGTVKENEDYILQFAPPFARLELLQANSCPDVIADHV 339

AFADEVLCWLYGTVKENEDYILQF PF RLELLQA SCPD I+HV

Sbjct: 484 AFADEVLCWLYGTVKENEDYILQFVHPFMRLELLQAQSCPDAITNHV 530

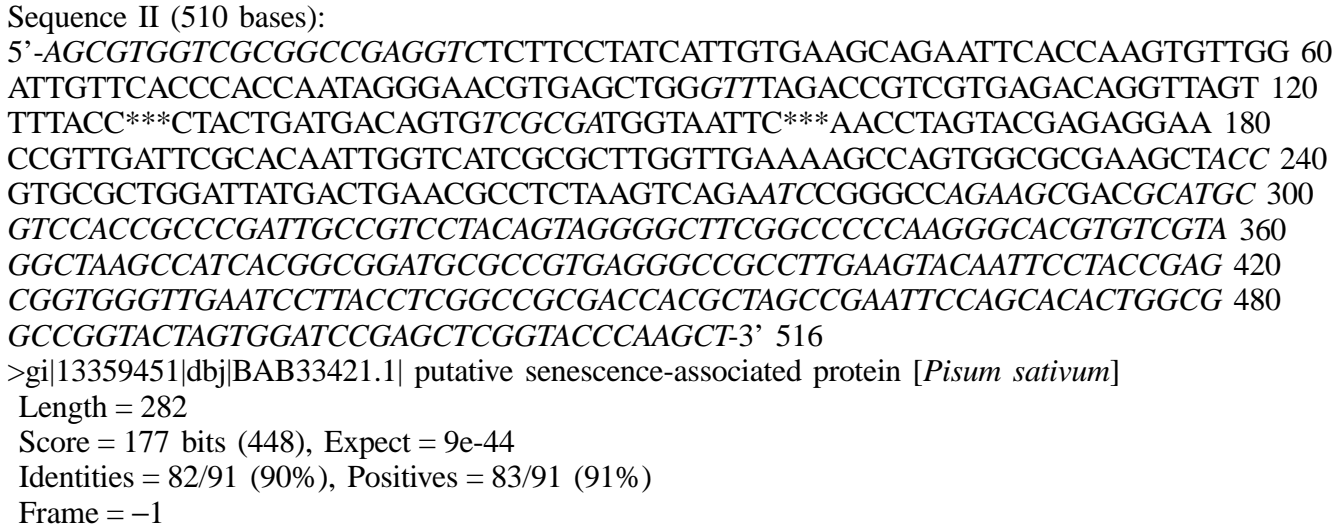

Fig. 4. Nucleotide sequences of Sequence I and Sequence II and their comparison with known sequences from GenBank non-redundant TRANSLATED query-PROTEIN databases [blastx]. Italic letters show the unique nucleotides of the sequences, *shows the deficient nucleotides.

metabolic changes (e.g. carbon metabolism, the synthesis of compatible solutes) and gene expression (such as aldose reductase homologue, genes responsing to abscisic acid induction etc) (Furini et al., 1997; Mundree et al., 2000; Xiong and Zhu, 2002; Zhang et al., 2002).

Based on its synthesis and accumulation fo osmoregulatory molecules and compounds during drought stress, $H$. ammodendron is assumed to belong to a group of angiosperms carrying out its drought tolerance mainly by physiological metabolisms (Chen and Pan, 2000). In addition, the drought tolerance of $H$. ammodendron is distinct from that of resurrection plants, such as Xerophyta viscose and Craterostigma plantagineum etc, because $H$. ammodendron doesn't possess drought tolerance at the initial stage of life, and it requires sufficient water to maintain regular growth and development during the period of germination and seedling. Only after this early drought-sensitive period does it become tolerant to drought stress (Huang, 1990). Therefore, if $H$. 
ammodendron exhibits responses at the molecular level to drought stress at an initial stage of the growth, this should be an event well worth studying.

Under natural conditions, high salinity and drought are the major causes of osmotic stress to plants. In our experiment here, by constructing the subtracted cDNA library, some specific cDNA clones of $H$. ammodendron seedlings induced by osmotic stress have been successfully isolated and identified; and 2 novel cDNA sequences putatively related with drought tolerance were obtained (Fig. 4). The results suggested that at the early growth and developmental stage, when encountering osmotic stress, the xerophyte $H$. ammodendron had rapid and abundant regulating activities at the gene expression level.

With the completion of the genome sequencing of Arabidopsis thaliana (The Arabidopsis Genome Initiative, 2000) and rice (Oryza sativa L.) (Goff et al., 2002; Yu et al., 2002), studies on plant functional genomics will lead to a dramatic increase in our knowledge about complex biological networks and the global impact of environmental conditions, such as drought, on the plant gene expression profile. Identification of full cDNA sequences or genes of $H$. ammodendron specific to osmotic stress will be completed in our future work. Drought tolerance genes have also being isolated from the resurrection plant (Craterostigma plantagineum) and the severe desiccation tolerant moss Physcomitrella by several international groups (Moffat, 2002). These achievements will help scientists to improve many plants, and especially crops, by achieving sustainable drought tolerance with the aid of genetic engineering techniques in the future.

Acknowledgments We are exceptionally grateful to the following programs who funded this research, The National 863 Pre-research Program of China, Fukang Observing Laboratory Station to Desert Ecological System of Xinjiang of the Chinese Academy of Sciences and The Foundation of Natural Sciences of Hunan Province (03 JJY 3026).

\section{References}

Bartels, D., Schneider, K., Terstappen, G., Piatkowski, D. and Salamini, F. (1990) Molecular cloning of abscisic acid modulated genes which are induced during desiccation of the resurrection plant Craterostigma plantagineum. Planta 181, 2734.

Bedunah, D. J. and Schmidt, S. M. (2000) Rangelands of Gobi Gurvan Saikhan National Conservation Park, Mongolia. Rangelands 22, 18-24.

Bohnert, H. J. and Sheveleva, E. (1998) Plant stress adaptations, making metabolism move. Curr. Opin. Plant Biol. 1, 267-274.

Chen, P. and Pan, X. L. (2000) Change of betaine contents and activity of betaine aldehyde dehydrogenase in seedlings of Haloxylon ammodendron under drought and $\mathrm{NaCl}$ stress.
Commun. Plant Physiol. 37, 520-522.

Diatchenko, L., Lau, Y. F. C., Campbell, A. P., Chenchik, A., Moqadam, F., Huang, B., Lukyanov, S., Lukyanov, K., Gurskaya, N., Sverdlov, E. D., and Siebert, P. D. (1996) Suppression subtractive hybridization: A method for generating differentially regulated or tissue-specific cDNA probes and libraries. Proc. Nat. Acad. Sci. USA 93, 6025-6030.

Furini, A., Koncz, C., Salamini, F. and Bartels, D. (1997) High level transcription of a member of a repeated gene family confers dehydration tolerance to callus tissue of Craterostigma plantagineum. EMBO J. 16, 3599-3608.

Goff, S. A., Ricke, D. and Lan, T. H. et al. (2002) A Draft Sequence of the Rice Genome (Oryza sativa L. ssp. japonica). Science 296, 92-100.

Hasegawa, P. M., Bressan, R. A., Zhu, J. K. and Bohnert, H. J. (2000) Plant cellular and molecular responses to high salinity. Annu. Rev. Plant Physiol. Plant Mol. Biol. 51, 463-499.

Huang, P. Y. (1990) Study on the restrictive factors affecting natural distribution of some xerophytic wood species in the desert of Xinjiang. Resources and Environment in Arid Regions 4, 59-67.

Ingram, J. and Bartels, D. (1996) The molecular basis of dehydration tolerance in plants. Annu. Rev. Plant Physiol. Plant Mol. Biol. 47, 377-403.

Moffat, A. S. (2002) Finding new ways to protect drought-stricken plants. Science 296, 1226-1229.

Mundree, S. G., Whittaker, A., Thomson, J. A. and Farrant, J. M. (2000) An aldose reductase homolog from the resurrection plant Xerophyta viscose Baker. Planta 211, 693-700.

Pan, X. L., Jiang, X. C., Guo, X. H., and Jiang Z. X. (2000) Study on the sensitivity of $H$. ammodendron (Mey.) Bge and O.sativa L. to responding to osmotic stress and exogenous $\mathrm{ABA}$ at the initial stage of seed germination. Seed 3, 16-18.

Pariasca, J. A. T., Sunaga, A., Miyazaki, T., Hisaka, H., Sonoda, M., Nakagawa, H. and Sato, T. (2001) Cloning of cDNAs encoding senescence-associated genes, ACC synthase and ACC oxidase from stored snow pea pods (Pisum sativum L. var saccharatum) and their expression during pod storage. Postharvest Biol. Technol. 22, 239-247.

Sambrook, J., Fritch, E. E. and Maniatis, T. (1989) Molecular Cloning: A Laboratory Manual, 2nd ed., pp. 362-371, Cold Spring Harbor Laboratory Press, New York, USA.

The Arabidopsis Genome Initiative. (2000) Analysis of the genome sequence of the flowering plant Arabidopsis thaliana. Nature 408, 796-815.

Xiong, L. and Zhu, J. K. (2002) Molecular and genetic aspects of plant responses to osmotic stress. Plant, Cell Environ. 25, 131139.

Yu, J., Hu, S. N. and Wang, J. et al. (2002) A Draft Sequence of the Rice Genome (Oryza sativa L. ssp. indica). Science 296, 79-92.

Zhang, X. N., Qu, Z. C., Wan, Y. Z., Zhang, H. W. and Shen, D. L. (2002) Application of suppression subtractive hybridization (SSH) to cloning differentially expressed cDNA in Dunaliella salina (chlorophyta) under hyperosmotic shock. Plant Mol. Biol. Reporter 20, 49-57.

Zhu, G. L., Mosyakin, S. L. and Clemants, S. E. (2003) Chenopodiaceae. Flora 5, 351-414. 